\title{
ON UNBOUNDED POLYNOMIAL DYNAMICAL SYSTEMS
}

\author{
Hamza BoujemaA And Said El Qotbi \\ Mohammed V University in Rabat, Morocco
}

\begin{abstract}
Suppose given a polynomial dynamical system of degree $m$. It is known that if the algebra associated to the corresponding homogeneous dynamical system of degree $m$ has an idempotent element then the original dynamical system is unbounded. In this work, we give a sufficient condition ensuring the unboundedness even when there is no idempotent. Some applications are also given.
\end{abstract}

\section{INTRODUCTION}

Since the seminal work of Markus [6], we can associate to any quadratic homogeneous system of differential equations in $\mathbb{R}^{n}$ a commutative but not necessarily associative multiplication in $\mathbb{R}^{n}$. If the system writes

$$
\dot{x}=P(x),
$$

where $x=\left(x_{1}, . ., x_{n}\right) \in \mathbb{R}^{n}$ and $P: \mathbb{R}^{n} \rightarrow \mathbb{R}^{n}$ is a function whose components are homogeneous quadratic polynomials in the variables $\left(x_{1}, . ., x_{n}\right)$, then the multiplication is a billinear symmetric map $\beta: \mathbb{R}^{n} \times \mathbb{R}^{n} \rightarrow \mathbb{R}^{n}$ defined by

$$
\beta(x, y)=\frac{1}{2}(P(x+y)-P(x)-P(y)) .
$$

In their paper on nonassociatice algebras and homogeneous quadratic differential equations (see [5]), Kinyon and Sagle gave substantial results and, more recently, this approach has been used for studying stability questions (see [1]).

2010 Mathematics Subject Classification. 34A34, 17A99.

Key words and phrases. Polynomial dynamical systems, homogenization, unboundedness. 
This process can be generalized to homogeneous polynomial differential equations of degree $m \geq 2$. In this case, an $m$-linear symmetric map

$$
f: \mathbb{R}^{n} \times \cdots \times \mathbb{R}^{n} \rightarrow \mathbb{R}^{n}
$$

can be defined and a $m$-algebra can be associated (see the work of Walcher $[7])$. We write in this case

$$
\dot{x}=f(x, . ., x)=x^{m} .
$$

An element $e \in \mathbb{R}^{n}$ different from the origin is said to be an idempotent if it verifies $e^{m}=f(e, . ., e)=e$ and an element $g \in \mathbb{R}^{n}$, also different from the origin, is called a nilpotent if we have $g^{m}=0$.

Among many interesting questions in the theory of differential equations, one can study the bounded corresponding vector fields (see [2]). A vector field is bounded if all trajectories are bounded for $t \geq 0$. For homogeneous polynomial differential equations, the existence of an idempotent ensures the unboundedness since the trajectory via this idempotent is a ray that blows up in finite time. Unfortunately, the existence of idempotents is not always given. In order to study the boundedness of polynomial differential equations of degree $m$, we try to gain informations from the study of the associated homogeneous polynomial differential equations of degree $m$ obtained from the initial one by dropping all terms of degree less than $m$. An easy to check sufficient condition was given by Dickson and Perko in [4]. Essentially, it says that if the corresponding nonassociative algebra (or $m$ - algebra when $m \geq 3$ ) has an idempotent then the polynomial vector field is unbounded.

From above, one can try to understand more when there is no idempotent. This fact has been emphasized by Coleman ([3]) who gave some results on unboundedness when the origin is an isolated critical point.

The aim of this work is to give a sufficient condition on the associated homogeneous polynomial differential system ensuring the unboundedness of the original polynomial dynamical system. This condition does not require the existence of idempotents. For the two dimensional case, in the second paragraph, we prove that if $L$ is a half line from the origin and if there exist, for the associated homogeneous polynomial dynamical system, a trajectory that conveniently crosses $L$ twice, then the polynomial dynamical system is unbounded. Using a geometrical approach based on Thales theorem, an elementary proof is given. Moreover, it is also shown that the blow up time is finite. The case of dimension 3 is studied in the third paragraph where we use an additional condition to ensure the iteration of some process and the same conclusions are obtained. Moreover, for dimension larger than 3, we emphasize similar conditions for a possible generalization. For dimension two, examples of odd degree are studied and for dimension three, two classes of examples are given. The first is of degree 2 and the second of degree 3 . 


\section{The two Dimensional CASE}

It is well known that, according to the homogenization process, if $X$ is a polynomial vector field on $\mathbb{R}^{n}$ of degree $m$ then we can associate to $X$ a homogeneous polynomial vector field $\hat{\mathrm{X}}$ of degree $m$ defined on $\mathbb{R}^{n+1}$. More precisely, if $\left(x_{1}, . ., x_{n}\right)$ are coordinates on $\mathbb{R}^{n}$ and $\left(x_{1}, . ., x_{n}, z\right)$ are coordinates on $\mathbb{R}^{n+1}$ and if $X$ writes

$$
\left\{\begin{array}{c}
\dot{x}_{1}=\sum_{1 \leq i_{1}^{1}+\cdots+i_{n}^{1} \leq m} a_{i_{1}^{1} \ldots i_{n}^{1}}^{1} x_{1}^{i_{1}^{1}} \ldots x_{n}^{i_{n}^{1}} \\
\vdots \\
\dot{x}_{n}=\sum_{1 \leq i_{1}^{n}+\cdots+i_{n}^{n} \leq m} a_{i_{1}^{1} \ldots i_{n}^{1}}^{n} x_{1}^{i_{1}^{n}} \ldots x_{n}^{i_{n}^{n}}
\end{array},\right.
$$

then $\hat{\mathrm{X}}$ writes

$$
\left\{\begin{array}{rl}
\dot{x}_{1} & =\sum_{1 \leq i_{1}^{1}+\cdots+i_{n}^{1} \leq m} a_{i_{1}^{1} \ldots i_{n}^{1}}^{1} x_{1}^{i_{1}^{1}} \ldots x_{n}^{i_{n}^{1}} z^{m-\left(i_{1}^{1}+\cdots+i_{n}^{1}\right)} \\
\vdots & \\
\dot{x}_{n} & =\sum_{1 \leq i_{1}^{n}+\cdots+i_{n}^{n} \leq m} a_{i_{1}^{1} \ldots i_{n}^{1}}^{n} x_{1}^{i_{1}^{n}} \ldots x_{n}^{i_{n}^{n}} z^{m-\left(i_{1}^{n}+\cdots+i_{n}^{n}\right)} \\
\dot{z} & =0
\end{array} .\right.
$$

We may notice that if we restrict $\hat{\mathrm{X}}$ to the hyperplane $\mathbb{R}^{n} \times\{z=1\}$, we obtain $X$ and if we restrict $\hat{\mathrm{X}}$ to $\mathbb{R}^{n} \times\{z=0\}$ we obtain the homogeneous part $\widetilde{X}$ of degree $m$ of $X$.

We state the main result.

Theorem 2.1. Let $X$ be a polynomial vector field of degree $m$ on $\mathbb{R}^{2}$ and $\widetilde{X}$ its homogeneous part of degree $m$. Let $\tilde{\phi}(\cdot, x)$ denotes the solution of $\tilde{X}$ through $x=\left(x_{1}, x_{2}\right) \in \mathbb{R}^{2}$. If there exist a half line $L$ from the origin $O$, a point $A \in L$ and two scalars $\tau>0, \lambda>1$ verifying $B=\tilde{\phi}(\tau, A)$ with $O B=\lambda O A$, then $X$ is unbounded and there is a trajectory blowing up in finite time.

Proof. If the trajectory through $A$ crosses the half line $L$ tangentially then, since $\widetilde{X}$ is homogeneous, $L$ is invariant and we have an idempotent. In this case, the result is given by Dickson and Perko in [4]. Thus, we may suppose that the trajectory meets $L$ transversely. We suppose also that $\tau$ is the smallest $t>0$ verifying $\phi(t, A) \in L$.

Let $\hat{\mathrm{X}}$ be the homogeneous polynomial vector field on $\mathbb{R}^{3}$ obtained from $X$ using the homogenization process. If we restrict $\hat{\mathrm{X}}$ to the hyperplane $z=0$, 
we obtain $\widetilde{X}$ the homogeneous part of $X$ and if we restrict it to the hyperplane $z=1$, in $\mathbb{R}^{3}$, we obtain $X$.

For $x=\left(x_{1}, x_{2}\right) \in \mathbb{R}^{2}$ and $\hat{x}=\left(x_{1}, x_{2}, z\right) \in \mathbb{R}^{3}$, if we let $\tilde{\phi}(\cdot, x)$ be the solution for $\tilde{X}$ through $x \in \mathbb{R}^{2}, \hat{\phi}(\cdot, \hat{x})$ be the solution for $\hat{X}$ through $\hat{x} \in \mathbb{R}^{3}$ and $\phi(\cdot, x)$ be the solution for $X$ through $x \in \mathbb{R}^{2}$, we have

$$
\hat{\phi}\left(t,\left(x_{1}, x_{2}, 1\right)\right)=\left(\phi\left(t,\left(x_{1}, x_{2}\right)\right), 1\right)
$$

and

$$
\hat{\phi}\left(t,\left(x_{1}, x_{2}, 0\right)\right)=\left(\tilde{\phi}\left(t,\left(x_{1}, x_{2}\right)\right), 0\right),
$$

when both sides are defined.

We consider the four points: $A=\left(a_{1}, a_{2}\right), B=\left(b_{1}, b_{2}\right), \hat{A}=\left(a_{1}, a_{2}, 0\right)$ and $\hat{B}=\left(b_{1}, b_{2}, 0\right)$. According to the hypothesis, since $\tilde{\phi}(\tau, A)=B$, we have $\hat{\phi}(\tau, \hat{A})=\hat{B}$.

Using the continuity of the flow $\hat{\phi}$, there exist a neighborhood $V(\hat{A}) \subset \mathbb{R}^{3}$ and a neighborhood $V(\hat{B}) \subset \mathbb{R}^{3}$ verifying

$$
\hat{\phi}(\tau, V(\hat{A})) \subset V(\hat{B}) .
$$

In addition, we may suppose $V(\hat{A}) \cap V(\hat{B})=\emptyset$ and we let $\gamma>0$ be the distance $d(V(\hat{A}), V(\hat{B}))$.

Let $\Pi$ be the two dimensional subspace of $\mathbb{R}^{3}$ containing the origin $O, \hat{A}$ and the vector $e_{3}=(0,0,1) \in \mathbb{R}^{3}$. As the vector field $\hat{X}$ is transverse to $\Pi$ at $\hat{B}$, it is possible to restrict $V(\hat{B})$ to a new neighborhood of $\hat{B}$, still denoted by $V(\hat{B})$, verifying: for any $y \in V(\hat{B})$, the trajectory through $y$ also meets transversely $\Pi$ at a point belonging to $\Pi \cap V(\hat{B})$. For the new neighborhood $V(\hat{B})$, the corresponding neighborhood of $\hat{A}$ will still be denoted by $V(\hat{A})$.

Let $\mathcal{B}(\hat{A}, \delta) \subset V(\hat{A})$ be an open ball centred in $\hat{A}$ and of radius $\delta>0$ small enough. First, we notice that if $y_{1} \in \mathcal{B}(\hat{A}, \delta)$ and $\tau_{y_{1}}$ is the smallest $t>0$ verifying

$$
\hat{\phi}\left(t, y_{1}\right) \in V(\hat{B}) \cap \Pi,
$$

then there exist two scalars $\tau_{1}, \tau_{2}$ with $0<\tau_{1}<\tau_{2}$ for which

$$
\tau_{1} \leq \tau_{y_{1}} \leq \tau_{2} \text { for any } y_{1} \in \mathcal{B}(\hat{A}, \delta)
$$

This follows from the compactness of the closed ball $\overline{\mathcal{B}(\hat{A}, \delta)}$ and the continuity of the map " $y_{1} \mapsto \tau_{y_{1}}$ ", following itself from the differentiability of some convenient Poincaré map.

Let $M_{1}=\left(a_{1}, a_{2}, \beta_{1}\right) \in \mathbb{R}^{3}$ with $\beta_{1}>0$ small enough so that $M_{1}$ belongs to $\mathcal{B}(\hat{A}, \delta)$. The line $\left(O M_{1}\right)$ crosses the hyperplane $z=1$ at a point $Q_{0}$. On the other hand, the point $N_{1}=\hat{\phi}\left(\tau_{M_{1}}, M_{1}\right)$ belongs to $V(\hat{B}) \cap \Pi$ and also to 
the hyperplane $z=\beta_{1}$. Consequently, the line $\left(O N_{1}\right)$ crosses the hyperplane $z=1$ at a point $Q_{1}$, belonging to $\Pi$ and verifying

$$
O Q_{1}=\frac{1}{\beta_{1}} O N_{1}
$$

See Figure 1.

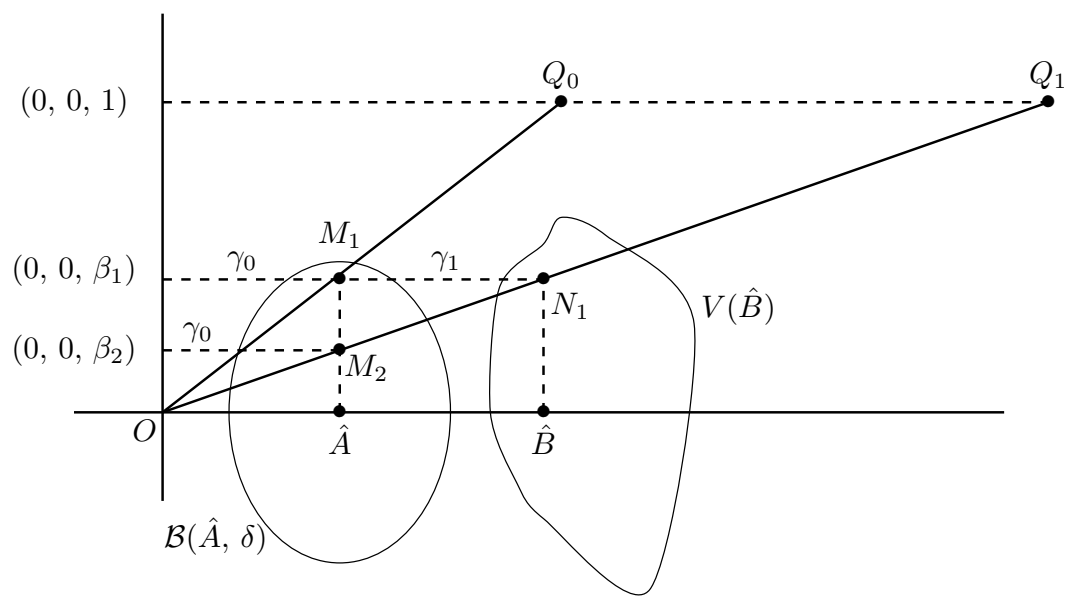

FiguRE 1.

For homogeneous quadratic dynamical systems in $\mathbb{R}^{n+1}$, it is known (see [5]) that the solution $\Phi$ verifies

$$
\Phi(t, a x)=a \Phi(a t, x)
$$

where $a$ is a scalar, $x \in \mathbb{R}^{n+1}$ and when both sides are defined. This implies that the trajectory through $a x$ and the trajectory through $x$ are homothetic. This fact can be generalized to homogeneous polynomial dynamical systems in $\mathbb{R}^{n+1}$ of degree $m \geq 2$ and we have

$$
\Phi(t, a x)=a \Phi\left(a^{m-1} t, x\right),
$$

when both sides are defined. As $Q_{0}$ is the homothetic of $M_{1}, Q_{1}$ is the homothetic of $N_{1}$ with respect to the same map and $N_{1}$ belongs to the trajectory of $M_{1}, Q_{1}$ also belongs to the trajectory of $Q_{0}$. As before, we can iterate the process. The line $\left(O Q_{1}\right)$ crosses the line $\left(\hat{A} M_{1}\right)$ at a point $M_{2}$ also element of $\mathcal{B}(\hat{A}, \delta) \cap \Pi$. If we let $M_{2}=\left(a_{1}, a_{2}, \beta_{2}\right)$ with $0<\beta_{2}<\beta_{1}$, then, using Thales theorem, we have

$$
\frac{\beta_{2}}{\beta_{1}}=\frac{\gamma_{0}}{\gamma_{0}+\gamma_{1}}<1
$$


where $\gamma_{1}=M_{1} N_{1}, \gamma_{0}$ being the distance from $M_{1}$ to the $z$-axis and also the distance from $M_{2}$ to the $z$-axis. We can notice that

$$
\frac{\beta_{2}}{\beta_{1}}<\frac{\gamma_{0}}{\gamma_{0}+\gamma}
$$

If we let $k=\frac{\gamma_{0}}{\gamma_{0}+\gamma}$, we can define by induction one sequence of positive scalars $\left(\beta_{n}\right)_{n}$ and three sequences of points $\left(M_{n}\right)_{n},\left(N_{n}\right)_{n}$ and $\left(Q_{n}\right)_{n}$ such that

$$
\beta_{n} \leq \beta_{1} k^{n-1}, Q_{n}=\frac{1}{\beta_{n}} N_{n}, Q_{n}=\frac{1}{\beta_{n+1}} M_{n+1}
$$

and

$$
Q_{n}=\frac{1}{\beta_{n}} \hat{\phi}\left(\tau_{M_{n}}, M_{n}\right)=\hat{\phi}\left(\beta_{n}^{m-1} \tau_{M_{n}}, \frac{1}{\beta_{n}} M_{n}\right)=\hat{\phi}\left(\beta_{n}^{m-1} \tau_{M_{n}}, Q_{n-1}\right) .
$$

Thus, we obtain

$$
Q_{n}=\hat{\phi}\left(\beta_{n}^{m-1} \tau_{M_{n}}+\cdots+\beta_{1}^{m-1} \tau_{M_{1}}, Q_{0}\right)
$$

and certainly $Q_{0} Q_{n} \geq n \gamma$, for $n \geq 1$. This shows that the trajectory for $\hat{X}$ through $Q_{0}=\left(q_{1}, q_{2}, 1\right)$ blows up and implies, using $(2.1)$, that the trajectory for $X$ through $\left(q_{1}, q_{2}\right)$ also blows up. Therefore, $X$ is unbounded. To justify that the blow up time is finite, we let $\xi_{n}$ be the scalar given by

$$
\xi_{n}=\beta_{n}^{m-1} \tau_{M_{n}}+\cdots+\beta_{1}^{m-1} \tau_{M_{1}} .
$$

Clearly, we have

$$
\begin{aligned}
\xi_{n} & \leq \tau_{2}\left(\beta_{n}^{m-1}+\cdots+\beta_{1}^{m-1}\right), \\
\xi_{n} & \leq \tau_{2}\left(\beta_{1}+\cdots+\beta_{n}\right), \\
\xi_{n} & \leq \tau_{2} \beta_{1} \frac{1}{1-k},
\end{aligned}
$$

which ends the proof.

EXAMPLE 2.2. We can notice that when dimension is two and if we expect a half line $L$ as in the theorem, the corresponding degree has to be odd.

Consider the following homogeneous polynomial dynamical system in $\mathbb{R}^{2}$

$$
\left\{\begin{array}{l}
\dot{x}=(-y+x)\left(x^{2}+y^{2}\right)^{k} \\
\dot{y}=(x+y)\left(x^{2}+y^{2}\right)^{k}
\end{array},\right.
$$

where $k$ is a positive integer.

The origin is obviously an isolated critical point and we can easily prove that there is no idempotent. In polar coordinates, system (2.3) writes

$$
\left\{\begin{array}{l}
\dot{r}=r^{2 k+1} \\
\dot{\theta}=r^{2 k}
\end{array}\right.
$$


Consequently, $r$ increases without bound and $\theta$ also increases. Thus trajectories spiral around the origin and get far away from it. For this example, any half line $L$ from the origin will work.

Using our theorem, any polynomial vector field under the form

$$
\left\{\begin{array}{l}
\dot{x}=(-y+x)\left(x^{2}+y^{2}\right)^{k}+P_{k}(x, y) \\
\dot{y}=(x+y)\left(x^{2}+y^{2}\right)^{k}+Q_{k}(x, y)
\end{array},\right.
$$

where $P_{k}$ and $Q_{k}$ are any polynomials of degree less than $2 k+1$, is unbounded.

\section{HigheR DIMENSIONS}

In this paragraph, we will use the same notations as before: $\mathrm{X}$ is a polynomial vector field of degree $m \geq 2$ on $\mathbb{R}^{n}$ for $n \geq 3, \tilde{X}$ its homogeneous part of degree $m$ and $\hat{X}$ the homogeneous vector field on $\mathbb{R}^{n+1}$ obtained from $X$ using the homogenization process. $\tilde{\phi}(., x)$ denotes the trajectory through a point $x=\left(x_{1}, . ., x_{n}\right) \in \mathbb{R}^{n}$ for $\tilde{X}$ and $\hat{\phi}(., \hat{x})$ the trajectory through $\hat{x}=\left(x_{1}, . ., x_{n}, z\right)$ for $\hat{X}$. We suppose that there exist two scalars $\tau>0$, $\lambda>1$ and a point $A \in \mathbb{R}^{n}$ such that, if $B=\tilde{\phi}(\tau, A)$ then $O B=\lambda O A$. If we consider the linear map $D \tilde{\phi}_{\tau}(A)$, we have the following result.

Proposition 3.1. $\lambda$ and $\lambda^{m}$ are two eigenvalues of $D \tilde{\phi}_{\tau}(A)$ and two corresponding eigenvectors are $u_{1}=A+(m-1) \tau \frac{\lambda}{1-\lambda^{m}} \tilde{X}(A)$ and $u_{2}=\tilde{X}(A)$.

Proof. Using the equality $\tilde{\phi}\left(\tau, \tilde{\phi}_{t}(A)\right)=\tilde{\phi}\left(t, \tilde{\phi}_{\tau}(A)\right)$ for small values of $t \in \mathbb{R}$, if we differentiate both sides with respect to $t$ and let $t=0$, we obtain

$$
D \tilde{\phi}_{\tau}(A)(\tilde{X}(A))=\tilde{X}\left(\tilde{\phi}_{\tau}(A)\right)=\tilde{X}(\lambda A)=\lambda^{m} \tilde{X}(A) .
$$

For the other eigenvalue, we notice that

$$
\tilde{\phi}(\tau,(1+t) A)=(1+t) \tilde{\phi}\left((1+t)^{m-1} \tau, A\right)
$$

for small values of $t \in \mathbb{R}$.

Again, if we differentiate with respect to $t$ and let $t=0$, we obtain

$$
D \tilde{\phi}_{\tau}(A)(A)=\lambda A+(m-1) \lambda^{m} \tau \tilde{X}(A),
$$

and if we look for a nonzero scalar $\beta$ for which

$$
D \tilde{\phi}_{\tau}(A+\beta \tilde{X}(A))=\lambda(A+\beta \tilde{X}(A)),
$$

we obtain $\beta=(m-1) \tau \frac{\lambda}{1-\lambda^{m}}$, which ends the proof.

REMARK 3.2. If $u=\left(u_{1}, u_{2}, u_{3}\right)$ is an eigenvector for $D \tilde{\phi}_{\tau}(A)$ then $\hat{u}=$ $\left(u_{1}, u_{2}, u_{3}, 0\right)$ is an eigenvector for $D \hat{\phi}_{\tau}(\hat{A})$ for the same eigenvalue as $u(\hat{A}$ is obtained from $A$ by adding 0 as a fourth component).

From now on, we suppose $n=3$ and we state the following result. 
TheOREM 3.3. Let $X$ be a polynomial vector field on $\mathbb{R}^{3}$ of degree $m, \tilde{X}$ its homogeneous part of degree $m$. We suppose that there exist $A \in \mathbb{R}^{3}$ and two scalars $\tau>0, \lambda>1$ verifying $\tilde{\phi}(\tau, A)=B$, with $O B=\lambda O A$ and we suppose also that the linear map $D \tilde{\phi}_{\tau}(A)$ has a third eigenvalue $\mu$ with $|\mu|<\lambda$ then $X$ is unbounded and there is a trajectory blowing up in finite time.

Proof. The main question is to make sure that an iteration process similar to the two dimensional case will be possible.

Let $\hat{X}$ be the homogeneous polynomial vector field on $\mathbb{R}^{4}$ obtained from $X, \hat{A} \in \mathbb{R}^{4}$ obtained from $A \in \mathbb{R}^{3}$ by adding 0 as a fourth component. Since coordinates will play an important role, we have to specify two convenient bases.

Starting from the canonical basis of $\mathbb{R}^{4}$, we let

$$
e_{1}=\frac{O \hat{A}}{\|O \hat{A}\|}, \quad e_{2}=\frac{\hat{X}(\hat{A})}{\|\hat{X}(\hat{A})\|}, \quad e_{3}=\frac{\hat{u}_{3}}{\left\|\hat{u}_{3}\right\|}, \quad e_{4}=(0,0,0,1) \text {, }
$$

where $\hat{u}_{3}$ is obtained from an eigenvector $u_{3}$ corresponding to the eigenvalue $\mu$, by adding 0 as a fourth component, and $\mathcal{B}_{1}$ is the basis given by $\mathcal{B}_{1}=$ $\left\{e_{1}, e_{2}, e_{3}, e_{4}\right\}$.

For any vector $w=\left(w_{1}, w_{2}, w_{3}, w_{4}\right)_{\mathcal{B}_{1}} \in \mathbb{R}^{4}$, we will adopt the norm

$$
\|w\|_{\mathcal{B}_{1}}=\operatorname{Sup}_{i=1, . ., 4}\left|w_{i}\right|
$$

and the coordinates of a point $M$ will be denoted in $\mathcal{B}_{1}$ by $\left(x_{M 1}, x_{M 2}, x_{M 3}, z_{M}\right)$.

We also introduce the basis $\mathcal{B}_{2}=\left\{E_{1}, E_{2}, e_{3}, e_{4}\right\}$ where $E_{1}=\frac{\hat{u}_{1}}{\left\|\hat{u}_{1}\right\|}$ and $E_{2}=\frac{\hat{u}_{2}}{\left\|\hat{u}_{2}\right\|}, \hat{u}_{1}$ and $\hat{u}_{2}$ are related respectively to $u_{1}$ and $u_{2}$ given in the previous proposition.

Similarly, we will denote the components of a point $M$ in $\mathcal{B}_{2}$ by $\left(X_{M 1}, X_{M 2}, x_{M 3}, z_{M}\right)$. In addition, we can notice that there exist a positive constant $c$ such that for any point $M \in \mathbb{R}^{4}$, we have $X_{M 1}=c x_{M 1}$. This fact will be used later.

Now, using properties of the flow, there exist a neighborhood $V(\hat{A}) \subset \mathbb{R}^{4}$ such that

$$
\hat{\phi}_{\tau}: V(\hat{A}) \rightarrow \hat{\phi}_{\tau}(V(\hat{A}))
$$

is a diffeomorphism.

If we let $H \subset \mathbb{R}^{4}$ be the hyperplane containing the line $(O \hat{A})$, parallel to $e_{3}$ and $e_{4}$, it is possible, using the transversality of $\hat{X}$ at $\hat{B}=\hat{\phi}_{\tau}(\hat{A})$, to restrict $V(\hat{A})$ to a new neighborhood still denoted by $V(\hat{A})$ and for which: for any $M \in V(\hat{A})$, the trajectory through $N=\hat{\phi}_{\tau}(M)$ meets $H$ at a point belonging to $H \cap \hat{\phi}_{\tau}(V(\hat{A}))$.

We also suppose that $V(\hat{A})$ is the ball $\mathcal{B}(\hat{A}, \delta)$ of radius $\delta>0$, small enough, the distance here is associated to the norm $\|\cdot\|_{\mathcal{B}_{1}}$. 
Without loss of generality, we may suppose $\mathcal{B}(\hat{A}, \delta) \cap \hat{\phi}_{\tau}(\mathcal{B}(\hat{A}, \delta))=\emptyset$ and we let

$$
\gamma=\operatorname{distance}\left(\mathcal{B}(\hat{A}, \delta), \hat{\phi}_{\tau}(\mathcal{B}(\hat{A}, \delta))\right)>0 .
$$

Let $M_{0} \in \mathcal{B}(\hat{A}, \delta)$ and $N_{0}$ be the point of $H \cap \hat{\phi}_{\tau}(\mathcal{B}(\hat{A}, \delta))$ at which the trajectory via $\hat{\phi}_{\tau}\left(M_{0}\right)$ crosses $H$.

We claim that if $\delta>0$ is small enough, the line $\left(O N_{0}\right) \operatorname{crosses} \mathcal{B}(\hat{A}, \delta)$ and the iteration is possible.

Clearly, in the basis $\mathcal{B}_{2}$, the matrix of $D \hat{\phi}_{\tau}(\hat{A})$ is

$$
\left(\begin{array}{cccc}
\lambda & 0 & 0 & 0 \\
0 & \lambda^{m} & 0 & 0 \\
0 & 0 & \mu & 0 \\
0 & 0 & 0 & 1
\end{array}\right)
$$

and there exist a point $P_{0} \in \mathcal{B}(\hat{A}, \delta)$ verifying $\hat{\phi}_{\tau}\left(P_{0}\right)=N_{0}$.

Let $N_{0}=\left(X_{N_{0} 1}, X_{N_{0} 2}, x_{N_{0} 3}, z_{N_{0}}\right), \hat{A}=\left(X_{\hat{A} 1}, X_{\hat{A} 2}, 0,0\right), \hat{B}=$ $\left(\lambda X_{\hat{A} 1}, \lambda X_{\hat{A} 2}, 0,0\right)$ and $P_{0}=\left(X_{P_{0} 1}, X_{P_{0} 2}, x_{P_{0} 3}, z_{P_{0}}\right)$, since

$$
\hat{\phi}_{\tau}\left(P_{0}\right)-\hat{\phi}_{\tau}(\hat{A})=D \hat{\phi}_{\tau}(\hat{A})\left(P_{0}-\hat{A}\right)+\left\|P_{0}-\hat{A}\right\|_{\mathcal{B}_{2}} \epsilon\left(P_{0}-\hat{A}\right),
$$

where $\epsilon=\left(\epsilon_{1}, \epsilon_{2}, \epsilon_{3}, \epsilon_{4}\right)$ is a function defined on a neighborhood of $0 \in \mathbb{R}^{4}$ with values in $\mathbb{R}^{4}$ verifying $\epsilon\left(P_{0}-\hat{A}\right) \rightarrow(0,0,0,0)$ when $\left\|P_{0}-\hat{A}\right\|_{\mathcal{B}_{2}} \rightarrow 0$, we can deduce the following system

$$
\left\{\begin{array}{l}
X_{N_{0} 1}-\lambda X_{\hat{A} 1}=\lambda\left(X_{P_{0} 1}-X_{\hat{A} 1}\right)+\left\|P_{0}-\hat{A}\right\|_{\mathcal{B}_{2}} \epsilon_{1}\left(P_{0}-\hat{A}\right) \\
X_{N_{0} 2}-\lambda X_{\hat{A} 2}=\lambda^{m}\left(X_{P_{0} 2}-X_{\hat{A} 2}\right)+\left\|P_{0}-\hat{A}\right\|_{\mathcal{B}_{2}} \epsilon_{2}\left(P_{0}-\hat{A}\right) \\
x_{N_{0} 3}=\mu x_{P_{0} 3}+\left\|P_{0}-\hat{A}\right\|_{\mathcal{B}_{2}} \epsilon_{3}\left(P_{0}-\hat{A}\right) \\
z_{N_{0}}=z_{P_{0}}+\left\|P_{0}-\hat{A}\right\|_{\mathcal{B}_{2}} \epsilon_{4}\left(P_{0}-\hat{A}\right)
\end{array} .\right.
$$

Because of the homogenization, we have $\epsilon_{4} \equiv 0$. Using a previous remark, if we introduce coordinates in $\mathcal{B}_{1}$, we deduce the system

$$
\left\{\begin{array}{l}
x_{N_{0} 1}=\lambda x_{P_{0} 1}+\left\|P_{0}-\hat{A}\right\|_{\mathcal{B}_{1}} \epsilon_{1}^{\prime}\left(P_{0}-\hat{A}\right) \\
x_{N_{0} 2}=0 \\
x_{N_{0} 3}=\mu x_{P_{0} 3}+\left\|P_{0}-\hat{A}\right\|_{\mathcal{B}_{1}} \epsilon_{3}^{\prime}\left(P_{0}-\hat{A}\right) \\
z_{N_{0}}=z_{P_{0}}
\end{array},\right.
$$

where $\epsilon_{1}^{\prime}, \epsilon_{3}^{\prime}$ are also functions defined on a neighborhood of $0 \in \mathbb{R}^{4}$ tending to $0 \in \mathbb{R}$ when $\left\|P_{0}-\hat{A}\right\|_{\mathcal{B}_{1}} \rightarrow 0$. The fact that we have $x_{N_{0} 2}=0$ results from the supposition $N_{0} \in H$.

In order to prove that the line $\left(O N_{0}\right)$ crosses $\mathcal{B}(\hat{A}, \delta)$, we need to find $\lambda^{\prime}>1$ for which the following inequalities hold

$$
\left|\frac{x_{N_{0} 1}}{\lambda^{\prime}}-x_{\hat{A} 1}\right|<\delta, \quad\left|\frac{x_{N_{0} 3}}{\lambda^{\prime}}\right|<\delta, \quad\left|\frac{z_{P_{0}}}{\lambda^{\prime}}\right|<\delta .
$$

The last inequality obviously holds since $P_{0} \in \mathcal{B}(\hat{A}, \delta)$. 
For the first inequality, using (3.1), it is equivalent to

$$
-\delta<\frac{\lambda}{\lambda^{\prime}} x_{P_{0} 1}-x_{\hat{A} 1}+\frac{\left\|P_{0}-\hat{A}\right\|_{\mathcal{B}_{1}}}{\lambda^{\prime}} \epsilon_{1}^{\prime}\left(P_{0}-\hat{A}\right)<\delta
$$

which leads to

$$
\frac{\lambda x_{P_{0} 1}+\left\|P_{0}-\hat{A}\right\|_{\mathcal{B}_{1}} \epsilon_{1}^{\prime}\left(P_{0}-\hat{A}\right)}{x_{\hat{A} 1}+\delta}<\lambda^{\prime}<\frac{\lambda x_{P_{0} 1}+\left\|P_{0}-\hat{A}\right\|_{\mathcal{B}_{1}} \epsilon_{1}^{\prime}\left(P_{0}-\hat{A}\right)}{x_{\hat{A} 1}-\delta} .
$$

On the other hand, the second inequality in (3.2) is equivalent to

$$
-\delta<\frac{\mu}{\lambda^{\prime}} x_{P_{0} 3}+\frac{\left\|P_{0}-\hat{A}\right\|_{\mathcal{B}_{1}}}{\lambda^{\prime}} \epsilon_{3}^{\prime}\left(P_{0}-\hat{A}\right)<\delta .
$$

As $\left|x_{P_{0} 3}\right|<\delta$ and $\left\|P_{0}-\hat{A}\right\|_{\mathcal{B}_{1}}<\delta$, it is enough to ask for

$$
\frac{|\mu|}{\lambda^{\prime}}+\frac{\left|\epsilon_{3}^{\prime}\left(P_{0}-\hat{A}\right)\right|}{\lambda^{\prime}}<1
$$

which means

$$
\lambda^{\prime}>|\mu|+\left|\epsilon_{3}^{\prime}\left(P_{0}-\hat{A}\right)\right| .
$$

Consequently, a sufficient condition for the existence of $\lambda^{\prime}$ is

$$
\frac{\lambda x_{P_{0} 1}+\left\|P_{0}-\hat{A}\right\|_{B_{1}} \epsilon_{1}^{\prime}\left(P_{0}-\hat{A}\right)}{x_{\hat{A} 1}-\delta}>|\mu|+\left|\epsilon_{3}^{\prime}\left(P_{0}-\hat{A}\right)\right| .
$$

Now as $x_{01}>x_{\hat{A} 1}-\delta$ and $\left\|P_{0}-\hat{A}\right\|_{\mathcal{B}_{1}}<\delta$, it is enough to verify that

$$
\lambda-\frac{\delta\left|\epsilon_{1}^{\prime}\left(P_{0}-\hat{A}\right)\right|}{x_{\hat{A} 1}-\delta}>|\mu|+\left|\epsilon_{3}^{\prime}\left(P_{0}-\hat{A}\right)\right| .
$$

If $\delta>0$ is small enough, then the functions $\epsilon_{1}^{\prime}$ and $\epsilon_{3}^{\prime}$ will be close enough to 0 and as $|\mu|<\lambda$, the condition (3.4) will hold. Consequently, it is possible to find $\lambda^{\prime}>1$ for which inequalities (3.2) are true. Thus, the claim is proved. Therefore, we can iterate. Given $\lambda^{\prime}>1$ verifying (3.2), we let $M_{1}=\frac{N_{0}}{\lambda^{\prime}}$, then the trajectory via $\hat{\phi}_{\tau}\left(M_{1}\right)$ meets $H \cap \hat{\phi}_{\tau}(\mathcal{B}(\hat{A}, \delta))$ at a point $N_{1}$ and the line $\left(O N_{1}\right)$ crosses $\mathcal{B}(\hat{A}, \delta)$ too. As for the two dimensional case, it is possible to define three sequences of points $\left(M_{n}\right)_{n \geq 0},\left(N_{n}\right)_{n \geq 0},\left(Q_{n}\right)_{n \geq 0}$. First, if we let $Q_{0}$ be the intersection point of the line $\left(O M_{0}\right)$ with the hyperplane " $z=1$ " and $Q_{1}$ the intersection point of the line $\left(O M_{1}\right)$ with the same hyperplane, $Q_{0}$ and $Q_{1}$ are elements of the same trajectory and we have

$$
\frac{Q_{0} Q_{1}}{M_{0} N_{0}}=\frac{1}{z_{M_{0}}}
$$

and similarly

$$
\frac{Q_{1} Q_{2}}{M_{1} N_{1}}=\frac{1}{z_{M_{1}}}
$$


If we consider the first components, we have

$$
x_{Q_{1} 1}-x_{Q_{0} 1}=\frac{1}{z_{M_{0}}}\left(x_{N_{0} 1}-x_{M_{0} 1}\right),
$$

and more generally, for $n \geq 1$

$$
x_{Q_{n} 1}-x_{Q_{n-1} 1}=\frac{1}{z_{M_{n-1}}}\left(x_{N_{n-1} 1}-x_{M_{n-1} 1}\right) .
$$

Since, for $n \geq 0, M_{n}$ and $N_{n}$ belong to two disjoint balls, we can deduce that

$$
x_{N_{n} 1}-x_{M_{n} 1} \geq \gamma
$$

and as

$$
x_{Q_{n} 1} \geq x_{Q_{n-1} 1}+\gamma \frac{1}{z_{M_{n-1}}},
$$

we have

$$
x_{Q_{n} 1} \geq x_{Q_{0} 1}+\gamma\left(\frac{1}{z_{M_{n-1}}}+\cdots+\frac{1}{z_{M_{0}}}\right)
$$

and therefore

$$
x_{Q_{n} 1} \geq x_{Q_{0} 1}+n \gamma .
$$

Consequently, when $n$ tends to $+\infty$, the trajectory blows up. To justify that the blow up time is finite, we recall that we have $N_{0}=\hat{\phi}_{\tau_{M_{0}}}\left(M_{0}\right)$ for some convenient scalar $\tau_{M_{0}}$ and more generally

$$
N_{n}=\hat{\phi}_{\tau_{M_{n}}}\left(M_{n}\right), n \geq 0
$$

In addition, there exist two scalars $\tau_{2}>\tau_{1}>0$ verifying

$$
\tau_{1} \leq \tau_{M_{n}} \leq \tau_{2}
$$

for any $n \geq 0$.

As $Q_{0}=\frac{1}{z_{M_{0}}} M_{0}$, we have

$$
Q_{1}=\frac{1}{z_{M_{0}}} N_{0}=\frac{1}{z_{M_{0}}} \hat{\phi}\left(\tau_{M_{0}}, M_{0}\right)=\hat{\phi}\left(z_{M_{0}}^{m-1} \tau_{M_{0}}, Q_{0}\right) .
$$

In the same way, we deduce

$$
Q_{n}=\hat{\phi}\left(z_{M_{n-1}}^{m-1} \tau_{M_{n-1}}+\cdots+z_{M_{0}}^{m-1} \tau_{M_{0}}, Q_{0}\right) .
$$

On the other hand, we have

$$
\frac{z_{M_{0}}}{z_{M_{1}}}=\frac{z_{N_{0}}}{z_{M_{1}}} \geq \frac{x_{\hat{A} 1}-\delta+\gamma}{x_{\hat{A} 1}+\delta}
$$

and more generally, for $n \geq 0$

$$
\frac{z_{M_{n-1}}}{z_{M_{n}}}=\frac{z_{N_{n-1}}}{z_{M_{n}}} \geq \frac{x_{\hat{A} 1}-\delta+\gamma}{x_{\hat{A} 1}+\delta} .
$$


If we define

$$
\xi_{n}=z_{M_{n-1}}^{m-1} \tau_{M_{n-1}}+\cdots+z_{M_{0}}^{m-1} \tau_{M_{0}} \text { and } k=\frac{x_{\hat{A} 1}+\delta}{x_{\hat{A} 1}-\delta+\gamma}
$$

then

$$
\begin{aligned}
& 0<\xi_{n} \leq \tau_{2}\left(z_{M_{n-1}}^{m-1}+\cdots+z_{M_{0}}^{m-1}\right) \\
& 0<\xi_{n} \leq \tau_{2} z_{M_{0}}^{m-1}\left(1+\cdots+k^{n-1}\right), \\
& 0<\xi_{n} \leq \frac{\tau_{2} z_{M_{0}}^{m-1}}{1-k}
\end{aligned}
$$

and the proof is complete.

REMARK 3.4. When the dimension is larger than 3 , as $\lambda$ and $\lambda^{m}$ are eigenvalues, a sufficient condition will be that the dimension of corresponding eigenspaces is 1 and that all other eigenvalues $\mu_{1}, . ., \mu_{p}$ have modulus less than $\lambda$. More explicitly, in the case where all eigenvalues are real and if $D \phi_{\tau}(A)$ is diagonalizable, there is no difficulty in proving the same result since we have

$$
\lambda>\operatorname{Sup}_{i=1, \ldots, p}\left|\mu_{i}\right| .
$$

If $D \phi_{\tau}(A)$ is not diagonalizable, then we use a Jordan canonical form for which all possible $1^{\prime} s$ above the main diagonal are replaced by some $\eta>0$ small enough to ensure

$$
\lambda>\operatorname{Sup}_{i=1, \ldots, p}\left|\mu_{i}\right|+\eta .
$$

When complex eigenvalues exist, similar ideas, with an appropriate choice of the norm to make the proof of the iteration easier, lead to the same result.

In the sequel, we will give two examples on $\mathbb{R}^{3}$, the first one is of degree 2 and the second is of degree 3 .

EXAmPle 3.5. Consider the following homogeneous polynomial dynamical system $\tilde{X}$ of degree 2

$$
\left\{\begin{array}{l}
\dot{x}=a x y+b y^{2}-c y z \\
\dot{y}=-a x^{2}-b x y+c x z+d y z \\
\dot{z}=d y^{2}
\end{array}\right.
$$

where $a, b, c$ and $d$ are real constants with $d>0$. Idempotents, if any, belong to the cone " $x^{2}+y^{2}=z^{2}$ ". If idempotents exist, unboundedness will result from Dickson and Perko theorem. Otherwise, we will deduce it from Theorem 3.3. Since

$$
\frac{d}{d t}\left(x^{2}+y^{2}-z^{2}\right)=0
$$

the cone " $x^{2}+y^{2}=z^{2} "$ is invariant. As $\dot{z}=d y^{2}$, if $(x(t), y(t), z(t))$ belongs to the cone, $z(t)$ increases without bound and, if we suppose that there is no 
idempotent, trajectories included in the cone will spiral and blow up. Thus, any generator of the cone is crossed infinitely often.

We let $A \neq 0$ be any point of the cone and $\tau>0$ the smallest scalar $t$ for which $\phi_{t}(A)$ crosses the generator $(O A)$. Since $D \tilde{\phi}_{\tau}(A)$ has $\lambda$ and $\lambda^{2}$ as eigenvalues, we have to justify that there exist a third eigenvalue $\mu$ such that $|\mu|<\lambda$. First, we may notice that the two eigenvectors $A+\frac{\lambda}{1-\lambda^{2}} \tau \tilde{X}(A)$ and $\tilde{X}(A)$ belong to the tangent plane to the cone at $A$. Moreover, as the cone is invariant, the third eigenvalue $\mu$ is necessarily positive. If we let $P_{1}(t)=(x(t), y(t), z(t))$ be a point inside the cone and if we consider the point

$$
P_{2}(t)=\left(z(t) \frac{x(t)}{\sqrt{x^{2}(t)+y^{2}(t)}}, z(t) \frac{y(t)}{\sqrt{x^{2}(t)+y^{2}(t)}}, z(t)\right),
$$

the point $P_{2}(t)$ belongs to the cone and $P_{1}(t), P_{2}(t)$ are elements of the same horizontal plane. Thus the distance $P_{1} P_{2}(t)$ represents the distance from $P_{1}(t)$ to its "horizontal projection" $P_{2}(t)$. On the other hand, since

$\frac{d}{d t} P_{1} P_{2}(t)=\frac{d}{d t}\left(z(t)-\sqrt{x^{2}(t)+y^{2}(t)}\right)=\frac{d y^{2}}{\sqrt{x^{2}+y^{2}}}\left(\sqrt{x^{2}+y^{2}}-z\right)<0$,

if we consider the map $\phi_{\tau}, \phi_{\tau}$ is contracting distances to corresponding horizontal projection points. As we may choose an eigenvector $u$ associated to $\mu$ pointing inward the cone, we can deduce that not only we have $|\mu|<\lambda$ but we have also $|\mu| \leq 1<\lambda$. According to our result, any polynomial vector field $X$ under the form

$$
\left\{\begin{array}{l}
\dot{x}=a x y+b y^{2}-c y z+P_{1}(x, y, z) \\
\dot{y}=-a x^{2}-b x y+c x z+d y z+Q_{1}(x, y, z) \\
\dot{z}=d y^{2}+R_{1}(x, y, z)
\end{array},\right.
$$

where $P_{1}, Q_{1}$ and $R_{1}$ are any linear functions defined on $\mathbb{R}^{3}$ with values in $\mathbb{R}$ with $P_{1}(0,0,0)=Q_{1}(0,0,0)=R_{1}(0,0,0)=0$, is unbounded.

EXAMPLE 3.6. Let $\tilde{Y}$ be the homogeneous polynomial system of degree 3 on $\mathbb{R}^{3}$ given by

$$
\left\{\begin{array}{l}
\dot{x}=a x^{2} y+b y^{3}+c x z^{2}+d y z^{2} \\
\dot{y}=-a x^{3}-b x y^{2}-d x z^{2} \\
\dot{z}=c x^{2} z
\end{array}\right.
$$

where $a, b, c$ and $d$ are real constants with $c>0$. Here again, the cone " $x^{2}+$ $y^{2}=z^{2} "$ is invariant and idempotents, if any, belong to the cone. As before, we suppose that there is no idempotent. In this case also, if $(x(t), y(t), z(t))$ belongs to the cone, then $z(t)$ increases without bound and the corresponding 


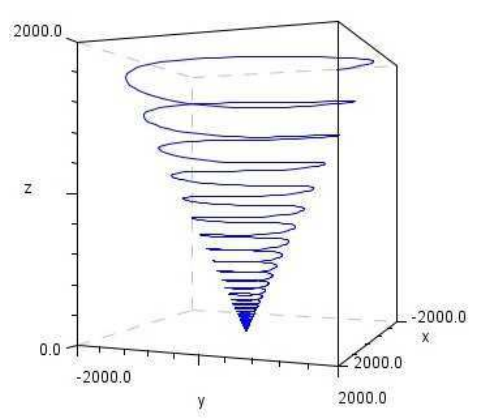

Figure 2. The cone for $m=2$

trajectory will spiral and blow up. Moreover, we have

$$
\frac{d}{d t}\left(z-\sqrt{x^{2}+y^{2}}\right)=\frac{c x^{2} z}{\sqrt{x^{2}+y^{2}}}\left(\sqrt{x^{2}+y^{2}}-z\right)<0
$$

inside the cone and this implies, as for the previous example, that the third eigenvalue $\mu$ verifies $\mu \leq 1<\lambda$. Thus, any polynomial vector field $X$ under the form

$$
\left\{\begin{array}{l}
\dot{x}=a x^{2} y+b y^{3}+c x z^{2}+d y z^{2}+P_{2}(x, y, z) \\
\dot{y}=-a x^{3}-b x y^{2}-d x z^{2}+Q_{2}(x, y, z) \\
\dot{z}=c x^{2} z+R_{2}(x, y, z)
\end{array},\right.
$$

where $P_{2}, Q_{2}$ and $R_{2}$ are three polynomials in the variable $x, y, z$ of degree less than 3 with $P_{2}(0,0,0)=Q_{2}(0,0,0)=R_{2}(0,0,0)=0$, is unbounded.

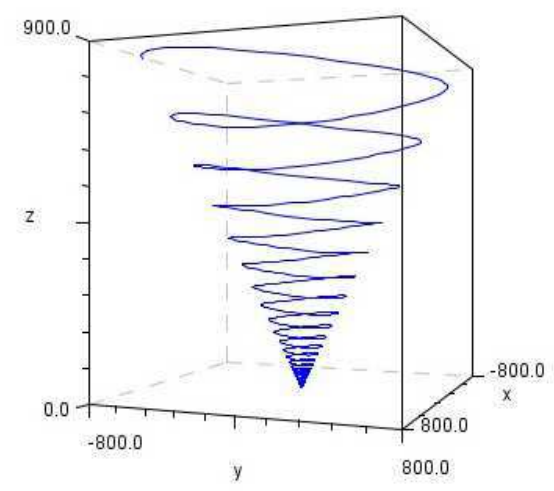

FiguRe 3 . The cone for $m=3$ 
ACKNOWLEDGEMENT.

The authors would like to thank the referee for the valuable remarks which helped to improve the content of this work.

\section{REFERENCES}

[1] H. Boujemaa, S. E. Qotbi and H. Rouiouih, Stability of critical points of quadratic homogeneous dynamical systems, Glas. Mat. Ser. III 51(71) (2016), 165-173.

[2] A. Cima and J. Llibre, Bounded polynomial vector fields, Trans. Amer. Math. Soc. 318 (1990), 557-579.

[3] C. S. Coleman, Boundedness and unboundedness in polynomial differential systems, Nonlinear Anal. 8 (1984), 1287-1294.

[4] R. J. Dickson and L. M. Perko, Quadratic differential systems, Lockheed Res. Rep. No. LMSC L-56-68-1, Lockheed Palo Alto Res. Lab, 1968.

[5] M. K. Kinyon and A. A. Sagle, Quadratic dynamical systems and algebras, J. Differential Equations 117 (1995), 67-126.

[6] L. Markus, Quadratic differential equations and non-associative algebra, in: 1960 Contributions to the theory of nonlinear oscillations, Vol. V, Princeton Univ. Press, Princeton, 1960, 185-213.

[7] S. Walcher, Algebras and differential equations, Hadronic Press, Palm Harbor, 1991.

\section{H. Boujemaa}

Département de Mathématiques

Mohammed V University in Rabat

1014RP Rabat

Morocco

E-mail: boujemaa@fsr.ac.ma

S. El Qotbi

Systèmes Dynamiques, A3D

Mohammed V University in Rabat

1014RP Rabat

Morocco

E-mail: Qotbis@gmail.com

Received: 24.11.2017. 